\title{
Aerodynamic simulation of evacuated tube maglev trains with different streamlined designs
}

\author{
Xuyong CHEN, Lifeng ZHAO*, Jiaqing MA, Yuansen LIU \\ Key Laboratory of Magnetic Levitation Technologies and Maglev Trains (Ministry of Education of China), Superconductivity and \\ New Energy R\&D Center, Southwest Jiaotong University, Chengdu 610031, China
}

\begin{abstract}
Based on the Navier-Stokes (N-S) equations of incompressible viscous fluids and the standard $k$ - $\varepsilon$ turbulence model with assumptions of steady state and two dimensional conditions, a simulation of the aerodynamic drag on a maglev train in an evacuated tube was made with ANSYS/FLOTRAN software under different vacuum pressures, blockage ratios, and shapes of train head and tail. The pressure flow fields of the evacuated tube maglev train under different vacuum pressures were analyzed, and then compared under the same blockage ratio condition. The results show that the environmental pressure of $1000 \mathrm{~Pa}$ in the tube is the best to achieve the effect of aerodynamic drag reduction, and there are no obvious differences in the aerodynamic drag reduction among different streamline head shapes. Overall, the blunt-shape tail and the blockage ratio of 0.25 are more efficient for drag reduction of the train at the tube pressure of $1000 \mathrm{~Pa}$.
\end{abstract}

Key words: aerodynamic drag; evacuated tube maglev train; blockage ratio; train head and tail

(C) 2012 JMT. All rights reserved.

\section{Introduction}

$\mathrm{T}$ he forthcoming energy shortage and the need to travel fast present many difficulties in the $21 \mathrm{st}$ century for traditional transportation, such as shipping, railway, waterway, and road transportation [1]. To solve these problems, Oster [2-3] proposed the concept of evacuated tube transportation (ETT). The ETT has numerous advantages such as high speed, low aerodynamic drag, low noise pollution, safety, less energy consumption, etc [4-6], and some solutions of the key vacuum technology for ETT have also been proposed [7].

Aerodynamics is a key issue for the application of evacuated tube maglev trains (ETMT) and therefore should be investigated thoroughly [8]. In recent years, several efforts of aerodynamic drag simulation were reported on a ETT train with a speed of $200 \mathrm{~m} / \mathrm{s}$ and a tube pressure of above $1000 \mathrm{~Pa}$ [9-10], and other efforts in numerical simulation of aerodynamic drag were reported on a subsonic ETT train with speed ranging from 50 to $300 \mathrm{~m} / \mathrm{s}$ and tube pressure from 10 to $10000 \mathrm{~Pa}$ [11]. Kwon et al. [12] concluded that the aerodynamic aspects must be included in the optimal design of the train nose, which is an improvement over

Received May 18, 2012; revision accepted Jun. 6, 2012

*Corresponding author. Tel.:+86-28-87600787

E-mail: zhaolf@home.swjtu.edu.cn (L.F.ZHAO)

(C) 2012 JMT. All rights reserved

doi: 10.3969/j.issn.2095-087X.2012.02.010 current design in terms of micropressure wave [13]. Streamline design of train head or tail and the related research has been conducted for traditional high-speed trains [14-16], metro trains [17], and maglev trains [1819]. However, little research has been done on the head and tail optimization of the ETMT.

In the present work, the external flow field of the maglev train with a speed of $300 \mathrm{~m} / \mathrm{s}$ in an evacuated tube was simulated with ANSYS/FLOTRAN software, based on the viscous fluid mechanics theory and the twodimensional incompressible viscous flow model. By considering different vacuum environments, three head types, and five tail types of trains for $0.11,0.14,0.18,0.25$, and 0.36 blockage ratios, we studied the relationships between the aerodynamic drag, shape of head and tail, and blockage ratio of the train. The results obtained may provide a helpful reference for future ETMT applications.

\section{Numerical model}

\subsection{Basic assumptions}

(1) The ETMT is assumed to run at a speed of $300 \mathrm{~m} / \mathrm{s}$ with a Mach number of 0.88 . As the Reynolds number of the flow field is greater than $10^{5}$, the flow field is considered turbulent, and the $k-\varepsilon$ two-equation turbulence model is used to simulate the flow field.

(2) The tube pressure of most models are assumed to be $1000 \mathrm{~Pa}$. Considering the computation time, conver- 
gence degree, computer performance, and blockage ratio, the gas is assumed to be incompressible. The tube temperature is assumed to be $293 \mathrm{~K}$.

(3) The simulation in this paper is dealt with in two dimensions. The largest longitudinal section of the maglev train is selected as the calculation plane, and the connection of the whole maglev train is assumed smooth.

(4) As the magnetic resistance is assumed to be negligible, only the aerodynamic drag is considered. According to the formula of aerodynamic drag, $F=\triangle P S$, the relationship between the aerodynamic drag $F$ and the pressure difference $\Delta P$ between the head and tail of the ETMT is linear, considering the largest cross-section area $S$ of the maglev train constant. Throughout this paper, the pressure difference $\Delta P$ means the difference between the maximum and minimum pressure values within the longitudinal scope of the train body from head to tail.

\subsection{Mathematical model}

The standard $k-\varepsilon$ turbulence model of the twodimensional viscous, steady and incompressible turbulent flow field is adopted to simulate the flow field of the ETMT. The control equations of the flow field mathematical model are as follows [20]:

continuity equation:

$$
\nabla \cdot \boldsymbol{U}=0
$$

conservation equations of momentum:

$$
\begin{aligned}
& \nabla \cdot(\rho u \boldsymbol{U})=\nabla \cdot(\mu \nabla u)-\frac{\partial p}{\partial x} \\
& \nabla \cdot(\rho v \boldsymbol{U})=\nabla \cdot(\mu \nabla v)-\frac{\partial p}{\partial y}
\end{aligned}
$$

turbulent kinetic energy equation:

$$
\begin{gathered}
\nabla \cdot(\rho \kappa \boldsymbol{U})=\frac{\partial}{\partial y}\left[\left(\mu_{1}+\frac{\mu_{t}}{\sigma_{k}}\right) \frac{\partial \kappa}{\partial y}\right]+ \\
\mu_{t}\left(\frac{\partial u}{\partial y}+\frac{\partial v}{\partial x}\right) \frac{\partial v}{\partial x}-\rho \varepsilon
\end{gathered}
$$

dissipation rate of turbulent kinetic energy equation:

$$
\begin{gathered}
\nabla \cdot(\rho \varepsilon \boldsymbol{U})=\frac{\partial}{\partial y}\left[\left(\mu_{1}+\frac{\mu_{t}}{\sigma_{\varepsilon}}\right) \frac{\partial \varepsilon}{\partial y}\right]+ \\
\frac{\varepsilon}{\kappa} C_{1} \mu_{t} \frac{\partial v}{\partial x}\left(\frac{\partial u}{\partial y}+\frac{\partial v}{\partial x}\right)-C_{2} \rho \frac{\varepsilon^{2}}{\kappa}
\end{gathered}
$$

where $\boldsymbol{U}$ is the flow field velocity vector, in $\mathrm{m} \cdot \mathrm{s}^{-1}, u$ and $v$ are the $x$ and $y$ components of $\boldsymbol{U}$, respectively; $p$ is the pressure of the flow field, in $\mathrm{Pa} ; \rho$ is the air density, in $\mathrm{kg} \cdot \mathrm{m}^{-3} ; \mu_{1}$ is the viscosity coefficient of laminar flow, in $\mathrm{N} \cdot \mathrm{s} \cdot \mathrm{m}^{-2} ; k$ is the turbulent kinetic energy, in $\mathrm{J} \cdot \mathrm{kg}^{-1} ; \varepsilon$ is the dissipation rate of the turbulence, in $\mathrm{J}^{\mathrm{kg}} \mathrm{kg}^{-1}$; and, the parameter $\mu_{t}$ is given by $\mu_{t}=\rho C_{\mu} k^{2} / \varepsilon$, where $C_{1}=1.47$, $C_{2}=1.92, C_{\mu}=0.09, \sigma_{k}=1.0$, and $\sigma_{\varepsilon}=1.3$.

\subsection{Longitudinal profile models of the maglev train}

In this research, different longitudinal profiles are designed for the ETMT, including three types of train heads and five types of tails, as shown in Fig. 1 and Fig. 2.

The basic parameters of the ETMT compartment are set as length $35 \mathrm{~m}$ and height $3 \mathrm{~m}$. The length of the rear and front portion will vary with the design style. For example, the entire train length is $41 \mathrm{~m}$ when it has a semicircle tail and a head with a radius of $3 \mathrm{~m}$, as shown in Fig. 3. Different streamlined designs of the tail also cause the train length to change (Fig. 2).

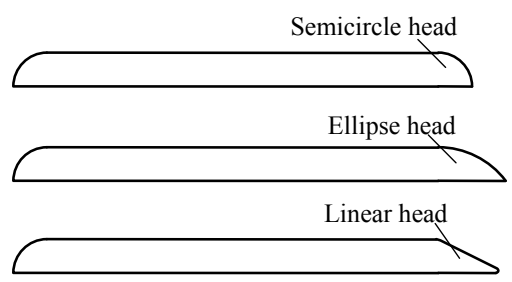

Fig. 1 Different models of ETMT head

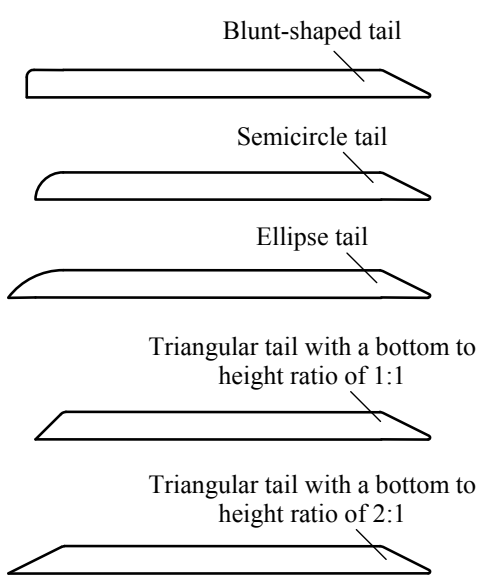

Fig. 2 Different models of ETMT tail

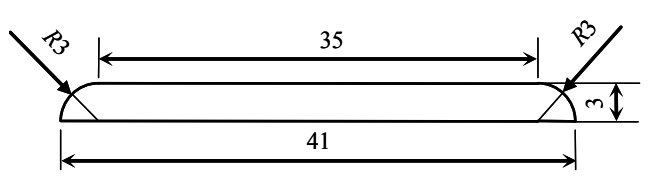

Fig. 3 Dimensions of the model train (unit: $\mathrm{m}$ ) 


\subsection{Simulation scenarios}

We developed simulations for three situations. The first is to analyze the influence of pressure on the ETMT under different vacuums. The second is to analyze three types of heads (Fig. 1) for the ETMT running under different blockage ratios. The third is to analyze the five types of tails (Fig. 2) for the ETMT running under a specific blocking ratio. Moreover, pressure differences of the blunt-shaped tail and the semicircle tail in different blocking ratios are compared according to the calculation results. For the second and third situations, the tube pressure is set as $1000 \mathrm{~Pa}$, and the ETMT speed is $300 \mathrm{~m} / \mathrm{s}$.

Using the ANSYS/FLOTRAN software, the flow pressure fields are simulated based on Eqs. (1) to (5) for the ETMT with different combinations of heads and tails, and the nephograms of ETMT in various situations are generated.

\subsection{Computational domain}

For the blockage ratios of $0.11,0.14,0.18,0.25$, and 0.36 , the tube diameter is set to be $9,8,7,6$ and $5 \mathrm{~m}$, respectively. One of the computational domain models is shown as an example in Fig. 4.

In Fig. 4, the entire tube length is $160 \mathrm{~m}$ and tube diameter $(6 \mathrm{~m}$, in this example) is set as the vertical height of the flow field. The total length of the ETMT chamber is $35 \mathrm{~m}$, the distance from the rear boundary of the train chamber to the inlet of the evacuated tube is $47 \mathrm{~m}$, and the distance from the front boundary of the train chamber to the outlet of the tube is $78 \mathrm{~m}$.

\subsection{Computing grid}

With the movement of the maglev train, moving meshing is used to deal with the constant change of the computational domain. The entire flow field area is divided into different sub-regions and separate meshes. The grids of various analytical model surfaces have a high density to ensure boundary layer calculation accuracy. The grids in the parts far away from the ETMT body, however, are not compacted in order to reduce the computation intensity and speed up convergence. For the situation shown in Fig. 4, a twodimensional meshing model is given in Fig. 5.

\section{Results and discussion}

Taking points of the pressure nephograms on the ETMT surface sequentially from tail to roof and then to head, we obtained pressure curves as shown in Figs. 6, 7, and 13. Figs. 8 to 12 illustrate the pressure nephograms of the ETMT with the five types of tails (Fig. 2), running under a specific blocking ratio.

\subsection{Pressure difference under different vacuums}

Fig. 6 shows the pressure difference under different vacuums of the train with semicircle tail and linear head (Fig. 4). We can see that the effect of drag reduction on the maglev train is negligible when the vacuum pressure is less than $1000 \mathrm{~Pa}$. As the vacuum pressure increases from 1000 to $10000 \mathrm{~Pa}$, the drag reduction effect enhances slowly, and then increases rapidly beyond $10000 \mathrm{~Pa}$. Thus the vacuum pressure of $1000 \mathrm{~Pa}$ seems the best for ETT operation.

\subsection{Pressure difference curves for different train heads}

Fig. 7 shows the maximum pressure difference curves of the ETMT with different heads (Fig. 1) running in blockage ratios of $0.11,0.14,0.18,0.25$, and 0.36 .

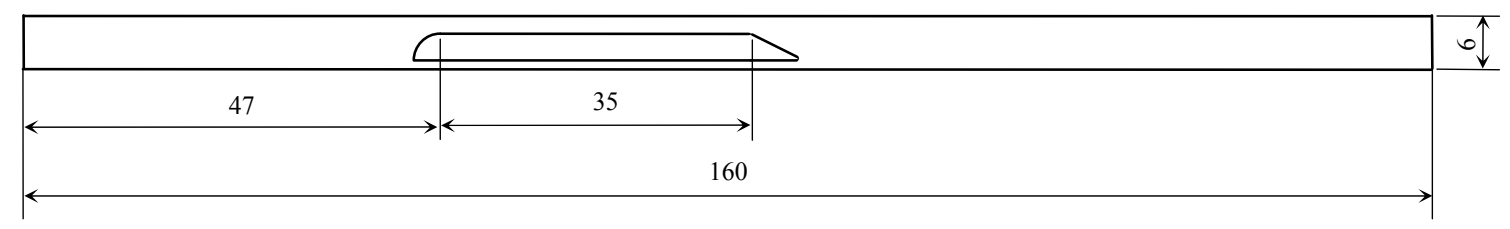

Fig. 4 Computational domain (unit: $\mathrm{m}$ )

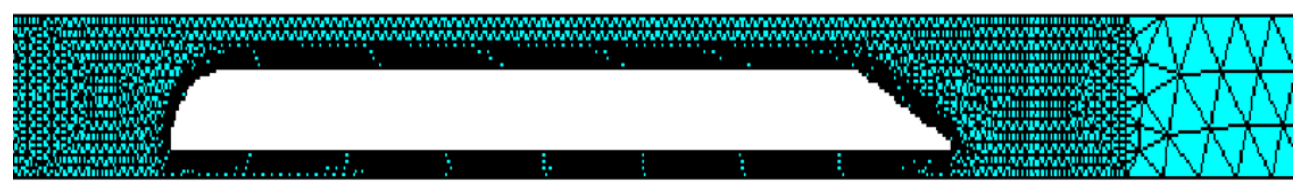

Fig. 5 Partial view of the meshing 
According to Fig. 7, the pressure differences for all head types increase with the blockage ratio. Furthermore, with the increase of blockage ratio, pressure difference increases slowly when the blockage ratio is less than 0.18 , and then increases sharply beyond the blockage ratio 0.18 . Meanwhile, we also notice that the pressure difference of the linear head is the smallest among the three head types, although their distinction is subtle.

Therefore, the drag reduction effect of the linear head is the best, and should be considered in the future

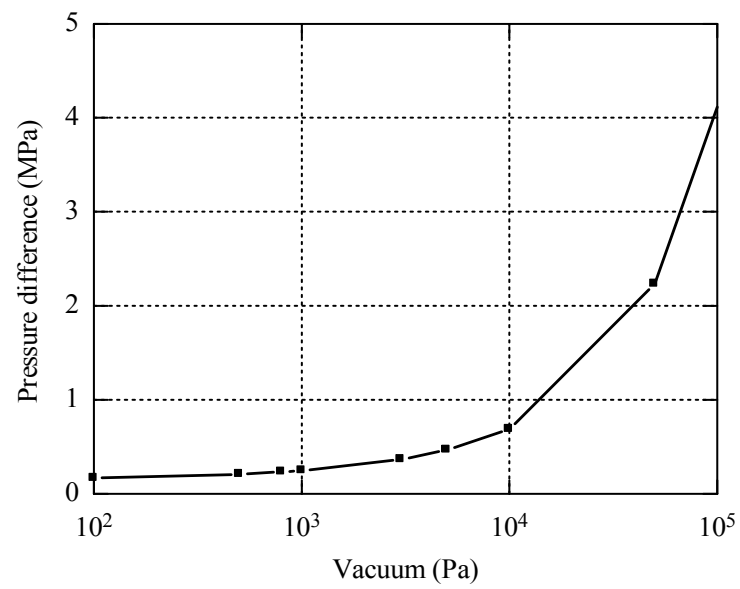

Fig. 6 Pressure difference under different vacuums application of the ETMT. In addition, the blockage ratio of 0.25 is more efficient at the tube pressure of $1000 \mathrm{~Pa}$.

\subsection{Pressure fields of different tails}

According to the results obtained above, we choose the linear head and a blockage ratio of 0.25 (tube diameter of $6 \mathrm{~m}$ ) to examine the effects of different tails on the pressure difference, while the other parameters remain unchanged. The calculated results are shown in Fig. 8 to Fig. 12.

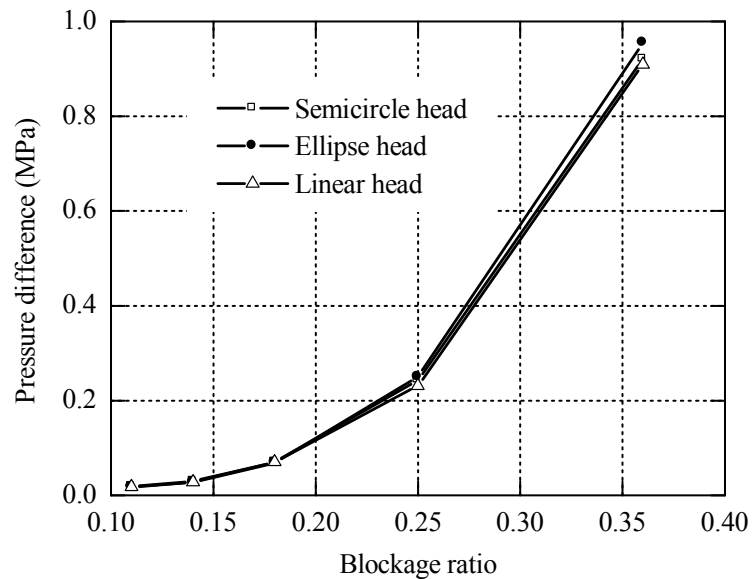

Fig. 7 Pressure difference vs. blockage ratio for different heads

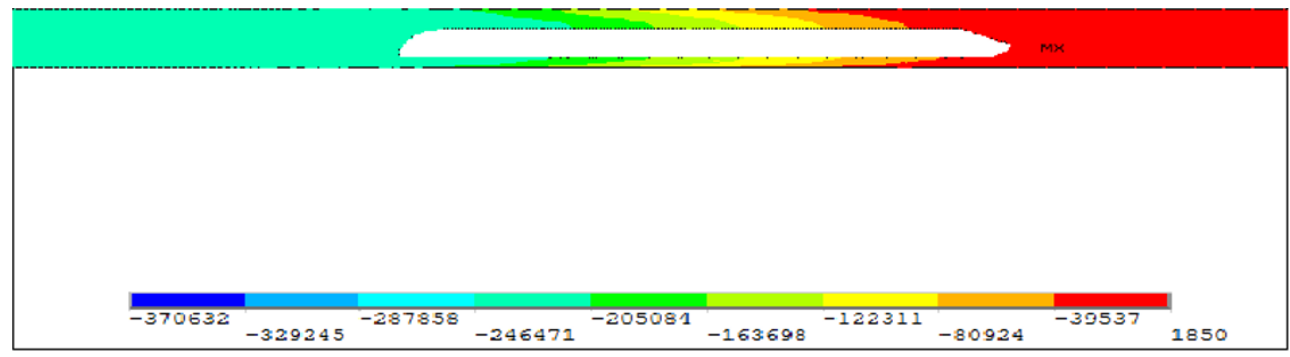

Fig. 8 Pressure field of the train with a semicircle tail

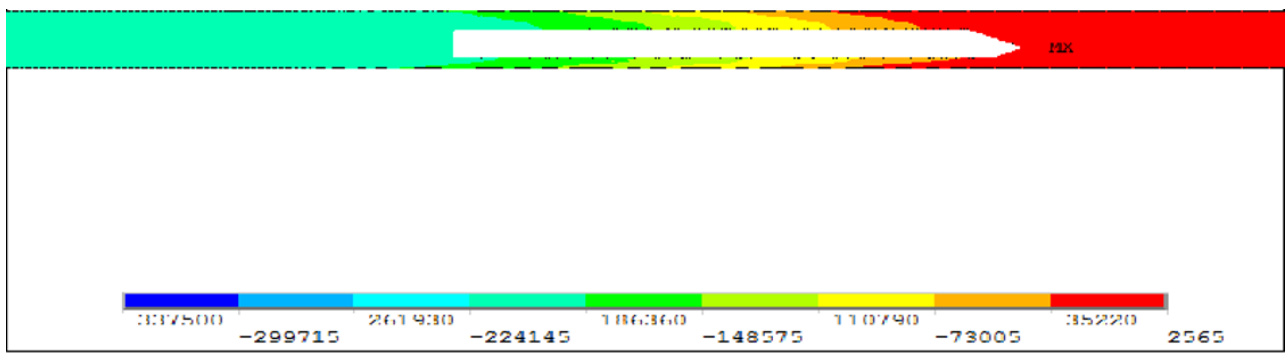

Fig. 9 Pressure field of the train with a blunt-shaped tail 


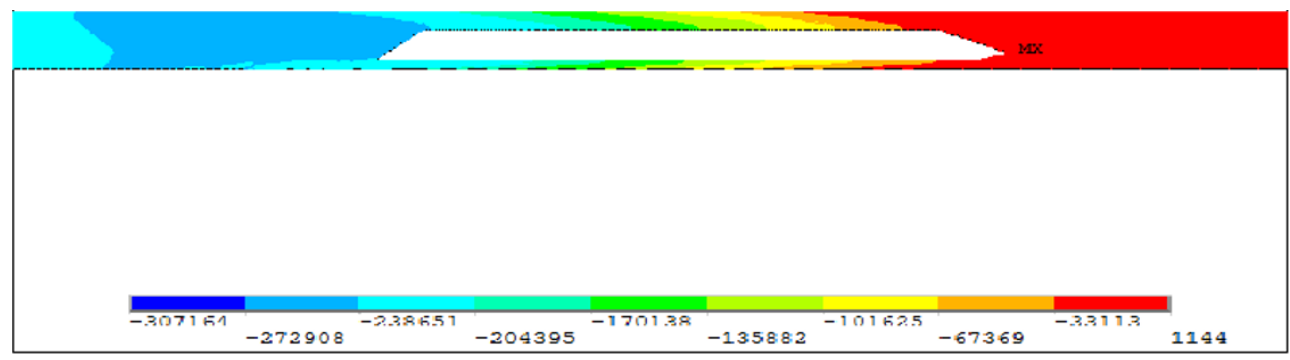

Fig. 10 Pressure field of the train with a triangular tail of 1:1 bottom to height ratio

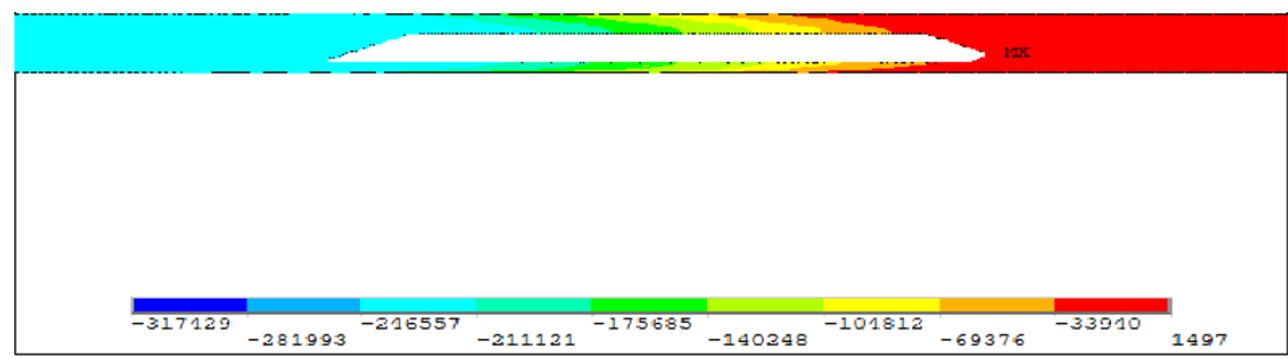

Fig. 11 Pressure field of the train with a triangular tail of 2:1 bottom to height ratio

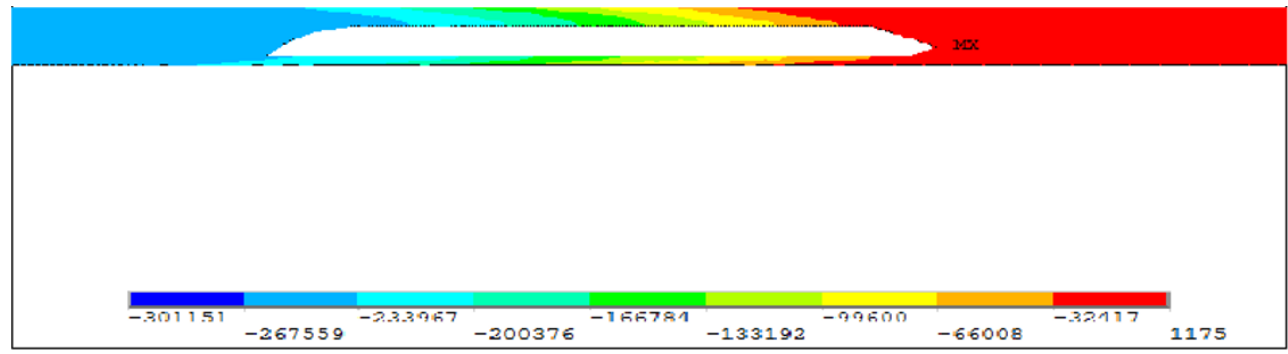

Fig. 12 Pressure field of the train with an ellipse tail

Table 1 Pressure difference $\Delta P$ with different tail shapes under the same blockage ratio of 0.25

\begin{tabular}{lc}
\hline \multicolumn{1}{c}{ Rear shape } & $\Delta P(\mathrm{kPa})$ \\
\hline Semicircle tail & 237.3 \\
Blunt-shaped tail & 204.8 \\
$\begin{array}{l}\text { Triangular tail with a bottom to } \\
\text { height ratio of 1: } 1\end{array}$ & 231.3 \\
$\begin{array}{l}\text { Triangular tail with a bottom to } \\
\text { height ratio of 2:1 }\end{array}$ & 244.5 \\
Ellipse tail & 241.3 \\
\hline
\end{tabular}

According to the pressure nephograms shown in Figs. 8 to 12 , the pressure distributions for the different tails are similar. A high pressure region, where the highest pressure point is marked with MX in each figure, can be observed on the surface of the train head. The pressure decreases gradually along the longitudinal direction of the maglev train, and even turns negative. We also find that among the five types of tails, the train with the blunt tail possesses the maximum pressure

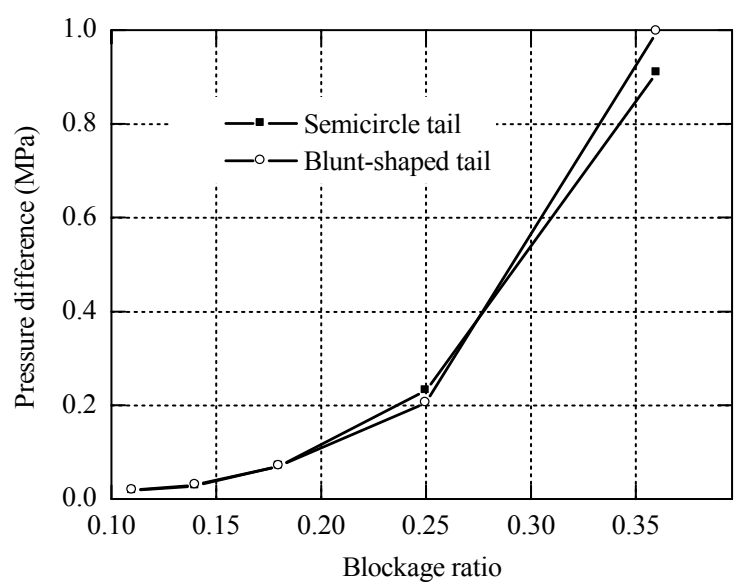

Fig. 13 Pressure difference vs. blockage ratio for semicircle tail train and blunt-shaped tail train

(2 $565 \mathrm{~Pa}$ ) on its head, while the pressure on the train head with a 1:1 bottom-height ratio triangular tail has the minimum value $(1144 \mathrm{~Pa})$. This means that the pressure value on the head is affected by the tail shape.

Fig. 13 shows the curves of the maximum pressure difference between head and tail varying with blockage 
ratio for linear head trains with blunt-shaped tail and semicircle tail. Pressure differences $\Delta P$ of the train with different tails is listed in Table 1. It is observed that the pressure difference of the maglev train with blunt-shape tail is apparently lower than others under the blockage ratio of 0.25 . However, under the blockage ratio of 0.36 , the pressure difference $\Delta P$ of the train with the blunt-shape tail is greater than that with the semicircle tail, as shown in Fig. 13. This reflects that the blockage ratio is also an important factor affecting the pressure difference value.

\section{Conclusions}

(1) The vacuum pressure plays a key role in the running drag of the ETMT. With the increase of vacuum pressure, the effect of the drag reduction increases very slowly when the pressure is less than $1000 \mathrm{~Pa}$, but rapidly when the pressure is above $10000 \mathrm{~Pa}$.

(2) The head with different streamlined designs does not cause obvious difference of aerodynamic drag effects for the maglev train running in the evacuated tube, but the linear head design can be regarded as one of the possible directions to reduce the aerodynamic drag of the ETMT in the future.

(3) The shape of tail exerts effects on the flow field around the train, causing the pressure on the train head and hence the pressure difference between head and tail to change, and this effect is also affected by blockage ratio.

\section{Acknowledgments}

This work was supported by the Program for Changjiang Scholars and Innovative Research Team in University (PCSIRT) of the Ministry of Education of China (IRT0751), the National High Technology Research and Development Program of China (863 program: 2007AA03Z203), the National Natural Science Foundation of China (Grant Nos. 50588201and 50872116), the Research Fund for the Doctoral Program of Higher Education of China (SRFDP200806130023), and the Fundamental Research Funds for the Central Universities (SWJTU09BR152, SWJTU09ZT24, and SWJTU11CX073).

\section{References}

[1] Y.P. Zhang, S.Z. Mei, X.G. Zeng, ETT-lead the highspeed transportation of the 21st century, World Sci-Tech Research and Development, 2002, 24(2): 60-64.

[2] D. Oster, ET3, http://www.et3.com, 2004-05-12.

[3] D. Oster, Crystal River, Fla. Evacuated Tube Transportation, United States Patent: 5950543, 1999.

[4] D. Oster, M. Kumada, Y.P. Zhang, Evacuated tube transport technologies $(\mathrm{ET} 3)^{\mathrm{tm}}$ : a maximum value global transportation network for passengers and cargo, Journal of Modern Transportation, 2011, 19(3):42-50.

[5] Y.P. Zhang, Y.Y. Li, Role and position of ETT in the future comprehensive transportation system, In: The Proceedings of International Conference on Transportation Engineering 2007, Chengdu, China, 2007: 2796-2803.

[6] Q.H. Qian, Some issues on design of underground magnetic levitation transportation system, Tunnel Construction, 2011, 31(2): 155-160 (in Chinese).

[7] Y.P. Zhang, D. Oster, M. Kumada, et al., Key vacuum technologies to be solved in evacuated tube transportation, Journal of Modern Transportation, 2011, 19(2): 110-113.

[8] Z.Y. Shen, On developing high-speed evacuated tube transportation in China, Journal of Southwest Jiaotong University, 2005, 40(2): 133-137 (in Chinese).

[9] X. Zhou, Y.P. Zhang, Y.F. Yao, Numerical simulation on the aerodynamic drag of high-speed train in evacuated tube, Science Technology and Engineering, 2008, 28(6): 1671-1819 (in Chinese).

[10] X. Zhou, D.Y. Zhang, Y.P. Zhang, Numerical simulation of blockage rate and aerodynamic drag of high-speed train in evacuated tube transportation, Chinese Journal of Vacuum Science and Technology, 2008, 28(6): 535-538 (in Chinese).

[11] Y.P. Zhang, Numerical simulation and analysis of aerodynamic drag on a subsonic train in evacuated tube transportation, Journal of Modern Transportation, 2012, 20(1): 42-50.

[12] H.B. Kwon, K.H. Jang, Y.S. Kim, et al., Nose shape optimization of high-speed train for minimization of tunnel sonic boom, JSME International Journal Series C Mechanical Systems, Machine Elements and Manufacturing, 2001, 44(3): 890-899.

[13] J.S. Lee, J.H. Kim, Approximate optimization of high-speed train nose shape for reducing micropressure wave, Structural and Multidisciplinary Optimization, 2008, 35(1): 79-87.

[14] J. Zhang, Research on optimum nose and tail shapes of foreign high-speed trains, Electric Drive for Locomotive, 2000, 35(2): 16-18 (in Chinese).

[15] R.L. Chen, Q.Y. Zeng, J. Xiang, et al., Study on the performance of aerodynamics of high-speed train with different nose shapes, Journal of Hunan University of Science and Technology (Natural Science Edition), 2009, 24(1): 45-48 (in Chinese).

[16] Y. Yan, W.N. Fang, Analysis of head shape design of high-speed train, In: Proceedings of the 2006 International Conference on Industrial Design and the 11th China Industrial Design Annual Meeting, Hangzhou, 2006: 152-156 (in Chinese).

[17] J.R. Zhang, L. Zhou, Q.H. Sun, et al., Simulated calculation of distributing of air flow around metro train head and its design analysis, Railway Locomotive and Car, 2008, 28(2): 43-47 (in Chinese).

[18] H.Q. Tian, D. Zhou, P. Xu, Aerodynamic performance and streamlined head shape of train, China Railway Science, 2006, 27(3): 47-55 (in Chinese).

[19] S.G. Yao, P. Xu, Aerodynamic shape optimization of domestic maglev train, Railway Locomotive and Car, 2007, 27(3): 33-34, 69 (in Chinese).

[20] H.Q. Tian, Train Aerodynamics, Beijing: China Railway Publishing House, 2007: 28-32 (in Chinese). 